\title{
Optimal Administrative Response to Selfish Behaviors in Urban Public Management: The Role of Zero-Determinant Strategies
}

\author{
Ai Zhong Shen $\mathbb{D},{ }^{1}$ Xiang Gao $\mathbb{D}^{2},{ }^{2}$ and Xiao Ping Wang $\mathbb{D}^{1}$ \\ ${ }^{1}$ Faculty of Professional Finance and Accountancy, Shanghai Business School, Shanghai, China \\ ${ }^{2}$ Research Center of Finance, Shanghai Business School, Shanghai, China \\ Correspondence should be addressed to Xiao Ping Wang; wangxiaoping0811@163.com
}

Received 11 August 2021; Accepted 30 September 2021; Published 18 October 2021

Academic Editor: Shaojian Qu

Copyright ( 2021 Ai Zhong Shen et al. This is an open access article distributed under the Creative Commons Attribution License, which permits unrestricted use, distribution, and reproduction in any medium, provided the original work is properly cited.

City management involves complex interactions between the manager (administrator), who supervises urban appearance and environmental sanitation, and the managed (speculator), who works in urban areas and is subject to management ordinances. This article provides an iterated game framework for analyzing the extent to which zero-determinant strategies can be used to optimize the intensity decision of supervisory action against municipal code violations, thus enhancing administrative efficiency. To account for characteristics of the public affairs context, it is assumed that each player in our model chooses from a finite set of discrete and random courses of game strategy. As our model constitutes a major extension to the seminal Press and Dyson (2012) model, we resort to the theory of stochastic process to prove the existence of multiple zero-determinant strategies when players can adopt many strategies in the iterated game. Various numerical examples are presented to validate such strategies' optimality. Our finding is that, given the probability of adopting a particular strategy, an urban administrator can unilaterally (i) set the speculators' expected payoff to a level equaling to the opportunity cost of abiding by the law and (ii) let their own expected surplus payoff exceed the speculators. Finally, important policy implications can be derived based on these analyses and conclusions.

\section{Introduction}

With the aiming of reducing the negative externality of individual behavior, urban public management involves using administrative inspection and supervision approaches to improve different aspects of urban public life including the environmental quality, city appearance, public health, and smoothness of traffic flow. The ideal practices for the management of urban areas can be accomplished through good cooperation between speculators exhibiting spontaneous law-abiding behaviors and the administrator performing monitoring duties without coercive enforcement. However, one always observes in the real world all kinds of selfish individual behaviors, which collectively can be coined the term "defection." Such defection behaviors caused various public management problems in Chinese cities such as the public safety concern caused by violations of traffic rules, the food safety issue when operating restaurants without sanitation or hygiene license, and the problem that unregistered vendors and street sellers may adversely affect the positive image of the city. Mitigating the conflict of ideal vs. reality in city administration has become a challenging task for urban public management officers. In the real world, selfish behaviors frequently occur on a large scale, which increases the costs of supervision and reduces the efficiency of public management. How to solve such a problem by devising an implementation path of city management regulations and, more importantly, how to determine the optimal strength of regulatory enforcement remain to be unanswered questions.

Many studies have attempted to address these questions from the perspective of the classic Nash equilibrium [1] and evolutionary game theory [2]. The research in applications of game theory concludes that defection will eventually happen in games like our urban administration setup and that selfish behavior exhibited by the majority of the population will disadvantage selfish speculators themselves as much as it will hurt those managers they are acting against. In practice, 
policy implementers often reduce the motivation to act selfishly by elevating supervision and intensifying punishment, which leads to significantly higher implementation costs and the occurrence of extreme events such as excessive enforcement or violent resistance. For example, city traffic managers often adopt the method of strengthening law enforcement, hoping to reduce traffic violations by punishing violators harsher and more frequent. But this method is not sustainable due to the high costs of maintaining strong enforcement. Once the strength of enforcement weakens, what often happens is that the illegal behaviors of drivers will return to the previous intensity. All city management efforts are expended in vain. Therefore, the broad questions raised at the end of the previous paragraph can be refined into a more purposeful yet crucial research direction. At what level should government officials set the supervision intensity so that urban public management can achieve a win-win situation, in which the altruistic behavior can be chosen voluntarily by the managed speculators? Due to the existence of payoff dynamics and many alternatives in the choice set, this refined question cannot be answered using merely the traditional game theory.

Therefore, we have constructed a new game theory model in which the players are the administrator and the speculator, and these two types of players can choose multiple different strategies according to varying supervision intensities for each round of the game. In this model, the player's choice of one strategy can be regarded as a Markov process, and there exists a state transferring probability matrix that determines the long-term payoffs of players in this iterated process. We use a random matrix to verify that the administrator can execute a linear mapping between the expected payoffs earned by both the administrator and the speculator through implementing a multistrategy zero-determinant strategy [3] (ZD strategy hereafter) in our model. We find that in the multistrategy iterated urban public management game, ZD strategies are still feasible. However, in contrast to the setting of a two-strategy game [3] or a multiplayer iterated game [4], the administrator player has multiple ZD strategies which are related to the number of strategies that can be chosen by the administrator in each round of the game. Equipped by these $\mathrm{ZD}$ strategies, the administrative body can unilaterally pin down the speculator's expected total payoff at a given level of the opportunity cost of law-abiding behavior. Under certain realistic conditions, our model suggests the government shift from increasing supervision intensity to providing public services that can reduce defection behaviors. Besides setting the opponents' total payoffs, administrators can enforce an extortionate linear relation between their own scores and the opponents' scores and can unilaterally ensure that their surplus turns out to be a discrete multiple of the surplus enjoyed by their opponents. Further, if the opposing speculators are rational individuals, the extortion strategy can promote spontaneous cooperative behavior undoubtedly.

Identifying ZD strategies for a multistrategy game matters for extending our understanding of the evolution of cooperation and provides a new perspective for achieving efficient urban public management. The study of a multistrategy game incorporating ZD strategies and the application of such a game to city administration are all research gaps that have yet been explored. The public environment of urban areas is a typical example of public goods. Rational individuals often exhibit defection behavior driven by shortterm interests, and as a result, the city's public environment becomes subject to the tragedy of the commons. However, either theoretical quantitative solution from existing microeconomic models or qualitative solution suggested by public management professionals seems to be unrealistic given the complicated choice set of game participants with unbalanced powers. Our proposed model is more in line with the practice because the administrator can adjust supervision strength according to the outcome ZD strategy, hence improving regulatory efficiency in the administrator's viewpoint and promoting potential violators to cooperate voluntarily. All in all, this paper enriches the literature on both ZD strategy game theory and urban public management theory as well as the relevant interdiscipline studies.

As a simple but complete model, we design the urban public management game to describe the decision of administrators about the optimal intensity of supervision, which depends on both the long-term game characteristics and the speculator's willingness to contribute so that the obvious tragedy of the commons can be avoided. Game theory has been proven to be critical for understanding, predicting, and intervening in many important public administration issues, ranging from smart grid pricing [5-7] to environmental pollution $[8,9]$ and fishery management [10]. Among several possible candidates, we believe that the iterated game serves as the most appropriate tool to provide insights into the abovementioned city administration issues. This is due to the extendibility of the iterated game as it can encompass all useful incentive mechanisms including reputation cooperation [11], kin selection cooperation [12], reciprocity cooperation [13], and reward and punishment [14-16]. Whether these mechanisms can elicit cooperative behavior is decided jointly by both sides of the game. However, without ZD strategy, the administrator cannot unilaterally determine the opponent's payoffs and lacks control over the implementation of these mechanisms. We, therefore, study the iterated urban public management game and apply a novel policy implementation mechanism based on the ZD strategy which empowers us with a strong ability to control the payoffs of speculators and improve their cooperation.

\section{Literature Review}

A clean and tidy urban environment is crucial for sustainable economic growth and better living conditions. The municipal government's regulations promulgated and decisions on how to enforce them would directly determine the efficiency of urban public management in securing the desired environment and attaining other indirect economic and social goals. Schwartz [17] reveals the importance of government capacity in enforcing a policy after investigating the cases for ten Chinese provinces. Hamm and Schrink [18] 
point out that the key factor of effective policy implementation is that the enforcement agency should possess sufficient management skills when exercising discretionary power. Kostka [19] believes that the dislocation of public sector functions and accountability has led to serious problems in terms of urban environmental governance. In sum, the regulatory ability, supervisory skills, and competence in duty assignments of municipal agencies represent different facets of government administration. These studies hence can provide solutions to our inefficiency problem of urban public management from merely the standpoint of administrators and via only the means of enhancing government administrative capability.

The incentives of government officials are also a key driver of successfully implementing urban environmental administration policies. Liu et al. [20] document that the structure of motivations designed for local government officials has become an institutional hurdle for environmental policy implementation and enforcement. For example, in China, government officials are more assessed by their performance regarding economic development. Therefore, much attention has been paid to GDP goals, whereas other policy objectives, such as urban public administration in this paper and environmental protection commonly seen in other studies, might be temporarily ignored in the short run. As the high-level government has the power to select politicians, local officials often choose to implement those policies that would please the upper government [21]. This also leads to conflicts between government officials and the general public [22]. In addition, the problem requires a much broader view concerning methodology since multiple actors are reacting strategically in policy implementation. On the one hand, it is difficult to enforce laws and regulations relying on only government bodies. The participation of the policy target group plays a large part here too. On the other hand, the complex policy environment and the shortcomings of the traditional bureaucratic structure make it hard for the government to fulfill tasks with limited resources. Therefore, this perspective, which is known as the policy network perspective, emphasizes the importance of modeling interactions between different actors [23]. The relevant empirical research shows that the participation of the residents of a city exerts a positive impact on urban public environmental policy implementation [24-26]. This strand of studies again highlights policy executors as the leading actor to solve public management problems. But it acknowledges that the policy-based network among the upper government, local officials, and the civil society is more conducive to the implementation of city management policy.

Next, the strength of policy implementation is also a key choice variable in solving the conflict between the administrator and the speculator to reduce the negative externality of defection behaviors. Prior research has pointed out that it is necessary to adopt campaign-style enforcement as an alternative governance mechanism to prevent policy implementation failure [27-29]. However, the campaign-style enforcement of public management policy cannot fundamentally solve the problem of public management under resource constraints. Another way of thinking is to treat the relationship between the city manager and speculators under management as a typical interactive decision game [30-32]. Under the analytical framework of game theory, government officials try their best to implement policies that they anticipate to result in a better public environment. However, selfish individuals constantly speculate to seek policy loopholes for satisfying their private interests, leading to serious negative externalities. The public administrator cannot unilaterally control opponents' payoffs by using traditional game strategies, nor induce speculators to adopt spontaneous cooperative behaviors. Suppressing selfish behaviors in such a collaborative relationship is crucial to turn the current situation around. Fortunately, Press and Dyson [3] introduced a new class of game strategies, i.e., the ZD strategies, for the two-player and two-strategy iterated prisoner's dilemma. Using this new strategy class, a player can unilaterally pin down his or her opponents' expected payoff or extort the opponent player by enforcing a linear relationship between his or her and the opponents' payoffs in the iterated prisoner's dilemma game. Follow-up studies on the ZD strategy [33-36] have reshaped our understanding of the traditional game and expanded the theoretical foundation of applying the game theory to various management fields. To put it in another way, the ZD strategies gives policy implementer a strong control ability, which can, in turn, guarantee the opponents to behave cooperatively. The ZD strategies can be naturally extended to provide solutions to multiplayer iterated games in the context of public goods games. The extant literature tells us that ZD strategies are still effective with many players: a single player is able to unilaterally determine the expected total payoffs for all other players in the multiplayer iterated game setting.

In this paper, we have put the idea of ZD strategy in the evolutionary game into practice in the field of urban public management. Our purpose is to optimize the supervision intensity decision made by the administrator during the process of city management policy implementation. Compared with traditional game theory applications in solving public management problems and in exploiting ZD strategy advantages, we make a distinction between our research and existing works in the following respects. First, we study the urban public administration issues by endogenizing the choice of policy implementation strength by the public environment administrator. Second, we emphasize that the public environment administrator can unilaterally determine the payoffs of speculators and hence bring about spontaneous cooperative behaviors. This capability improves the efficiency of public management. Third, we advance the application-oriented research of ZD strategies [3] to a more generalized stage. In specific, we have taken a step further by extending the two-strategy game to a multistrategy setting, which can be a big addition to deepen our understanding of cooperation evolution. Finally, we attempt to answer the pivotal but open question in urban public management: can government officials control the behavior of speculators and promote cooperation behavior through effective supervision mechanisms based on the ZD strategies instead of increasing the intensity of supervision and punishment? 


\section{The Model}

3.1. Basic Setup and Payoff Matrix. A typical evolutionary game includes three main elements: the players, the game strategy set, and the payoff function. The players of our urban public management game are the speculator $X$ and the administrator $Y$. The term "speculator" refers to individuals who engage in irregular or illegal activities to serve their self-interests, such as traffic offenders, unlicensed street vendors or peddlers, and enterprises polluting the natural environment. Let the "administrator" be a member of the government's corresponding administrative department, such as traffic police, a city management officer, and an environmental protection inspector.

The game strategy set includes alternative actions that can be taken by the players in each round of the game. For example, the players' strategy set comprises the cooperation and defection action in the famous prisoner's dilemma game. In our model, the speculator has two alternative actions, which are breaking the regulation $s_{X}(0)$ and obeying the regulation $s_{X}(1)$. We thus denote the strategy set of the speculator by $S_{X}=\left(s_{X}(0), s_{X}(1)\right)$. Turning to the other side, the administrator can take different actions according to different levels of supervision intensity, which constitutes an important decision variable in our model. The supervision intensity $K \in[0, k]$ is a discrete random variable and the strictness of the supervision increases with the value of $K$. As the administrator can adopt different action strategies with changes in $K$, the strategy set of the administration is denoted by

$$
S_{Y}=\left(s_{Y}(0), s_{Y}(1), s_{Y}(2), \ldots, s_{Y}(k)\right),
$$

where $s_{Y}(0)$ and $s_{Y}(k)$ represent nonsupervision and complete supervision, respectively. In the urban public management game, a player will choose a strategy from the set, and the strategy pair $(x, y)$ chosen by both players $X$ and $Y$ is a combination of strategies at each round of the game. When a certain action strategy combination is exogenously given, the result of the game can be expressed as the players' payoff function $u_{X}(x, y)$ and $u_{Y}(x, y)$.

The payoff function in city management is related to efficiency gains for the administrator; whereas it is about cost cuts for the speculator. The supervision intensity $K$ will affect both the implementation cost and the expected return of the administrator. Suppose that the total cost of implementation is $k \times c$ at the supervision intensity of $k$. The reputation gains from efficient public management and the expected fines collected by punishing illegal behavior of speculators are $r_{1}(k)$ and $r_{2}(k)$, respectively. We further assume that the cost to speculators comprises two parts: the punishment cost $r_{2}(k)$ and the opportunity cost $a$. The opportunity cost equals the sum of gains abandoned when the speculator chooses to conform to laws and regulations. For example, a street peddler who chooses to observe the law will have to forgo illegal income from unlicensed operations and go through complex bureaucratic procedures to establish a real business and pay due taxes. The expected gains from the illegal behavior of speculators are $R$. According to the cost- benefit analysis conducted above, the payoff matrix of both players under different strategy combinations is given in Table 1.

\subsection{Zero-Determinant Strategies in Multistrategy Games.} Consider a $k$-strategy iterated public management game led by the administrator, in which certain intermediate stage games between the speculator and the administrator are infinitely iterated. The existing literature points out that for any strategy of the longer-memory player $Y$, the shortermemory player $X$ 's score is exactly the same when the same game (same in the sense that the allowed moves and the payoff matrix are all identified) is indefinitely iterated. That is, a long-memory player has no advantage over shortmemory players. Therefore, in our urban public management game, we can assume that a player's strategy in the current round of the game depends only on the outcome produced by the previous round. For each round of the game, the two players under concern may choose their respective game strategy from their respective strategy sets. Thus, the possible outcomes obtained by the speculator $X$ with the strategy combination in each round of the game are

$$
\left(\begin{array}{lllll}
s_{X}(0) s_{Y}(0) & s_{X}(0) s_{Y}(1) & s_{X}(0) s_{Y}(2) & \ldots & s_{X}(0) s_{Y}(k) \\
s_{X}(1) s_{Y}(0) & s_{X}(1) s_{Y}(1) & s_{X}(1) s_{Y}(2) & \ldots & s_{X}(1) s_{Y}(k)
\end{array}\right) .
$$

These elements can be denoted as $s_{X}(i) s_{Y}(j)$, $i \in\{0,1\}, j \in\{0,1,2,3, \ldots, k\}$, where $k$ is a finite discrete random variable. For the speculator $X$, a mixed strategy $p_{i j}^{n}\left(0 \leq p_{i j}^{n} \leq 1, n \in\{0,1\}\right)$ is a vector that consists of conditional probabilities for each strategy $s_{X}(i)$. These probabilities are given, with respect to each of the possible game outcomes, as follows:

$$
p_{i j}^{n}=\left(p_{00}^{0}, p_{00}^{1}, p_{01}^{0}, p_{01}^{1}, \ldots, p_{0 k}^{0}, p_{0 k}^{1}, p_{10}^{0}, p_{10}^{1}, p_{11}^{0}, p_{11}^{1}, \ldots, p_{1 k}^{0}, p_{1 k}^{1}\right),
$$

where $p_{i j}^{n}$ represents the probability of strategy $s_{X}(n)$ in the current round conditioning on the previous round's results. The superscript and subscript of $p_{i j}^{n}$ represent the strategy combination of the previous game outcomes and the current game strategy of the speculator $X$, respectively. Take $p_{00}^{1}$ for example; it represents the probability that one simultaneously observes $s_{X}(1)$ being the present round game strategy and $\left(s_{X}(0), s_{Y}(0)\right)$ being the previous game outcome.

Similarly, the possible outcomes obtained by the administrator $Y$ in each round of the game can be given by

$$
\left[\begin{array}{cc}
s_{Y}(0) s_{X}(0) & s_{Y}(0) s_{X}(1) \\
s_{Y}(1) s_{X}(0) & s_{Y}(1) s_{X}(1) \\
s_{Y}(2) s_{X}(0) & s_{Y}(2) s_{X}(1) \\
\ldots & \ldots \\
s_{Y}(k) s_{X}(0) & s_{Y}(k) s_{X}(1)
\end{array}\right] .
$$


TABLE 1: The payoff matrix.

\begin{tabular}{|c|c|c|c|c|c|}
\hline & $S_{Y}(0)$ & $S_{Y}(1)$ & $S_{Y}(2)$ & $\cdots$ & $S_{Y}(k)$ \\
\hline$S_{X}(0)$ & $a, r_{1}(0)$ & $a, r_{1}(1)-c$ & $a, r_{1}(2)-2 c$ & . & $a, r_{1}(k)-k c$ \\
\hline$S_{X}(1)$ & $R-r_{2}(0), r_{2}(0)+r_{1}(0)$ & $R-r_{2}(1), r_{2}(1)+r_{1}(1)-c$ & $R-r_{2}(2), r_{2}(2)+r_{1}(2)-2 c$ & $\ldots$ & $R-r_{2}(k), r_{2}(k)+r_{1}(k)-k c$ \\
\hline
\end{tabular}

An element in the above outcome matrix can be abstracted as $s_{Y}(j) s_{X}(i), i \in\{0,1\}, j \in\{0,1,2,3, \ldots, k\}$. For the administrator $Y$, the conditional probabilities $q_{j i}^{m}$ $\left(0 \leq q_{j i}^{m} \leq 1, m \in\{0,1,2,3, \ldots, k\}\right)$ for the strategy $s_{Y}(j)$ with respect to each of these possible outcomes can be written as

$$
q_{j i}^{m}=\left(q_{00}^{0}, q_{00}^{1}, \ldots, q_{00}^{k}, q_{01}^{0}, q_{01}^{1}, \ldots, q_{01}^{k}, \ldots, q_{10}^{0}, q_{10}^{1}, \ldots, q_{10}^{k}, \ldots, q_{k 0}^{0}, q_{k 0}^{1}, \ldots, q_{k 0}^{k}, q_{k 1}^{0}, q_{k 1}^{1}, \ldots, q_{k 1}^{k}\right)
$$

where $q_{j i}^{m}$ represents the probability of strategy $s_{Y}(m)$ in the current round conditional on the output of the previous round game. That is, the probability of having a supervision intensity of $m$ is $q_{j i}^{m}$ in the current round, conditional on the outcome of the previous game round.
Since this study considers a memory-one iterated game, the public management game can be characterized by a Markov chain. Suppose that $P(p, q)$ is used to represent the state transition probability matrix of this Markov process.

$$
P(p, q)=\left[\begin{array}{ccccccccc}
p_{00}^{0} q_{00}^{0} & p_{00}^{0} q_{00}^{1} & \ldots & p_{00}^{0} q_{00}^{k} & p_{00}^{1} q_{00}^{0} & p_{00}^{1} q_{00}^{1} & \ldots & p_{00}^{1} q_{00}^{k} \\
p_{10}^{0} q_{01}^{0} & p_{10}^{0} q_{01}^{1} & \ldots & p_{10}^{0} q_{01}^{k} & p_{10}^{1} q_{01}^{0} & p_{10}^{1} q_{01}^{1} & \ldots & p_{10}^{1} q_{01}^{k} \\
p_{01}^{0} q_{10}^{0} & p_{01}^{0} q_{10}^{1} & \ldots & p_{01}^{0} q_{10}^{k} & p_{01}^{1} q_{10}^{0} & p_{01}^{1} q_{10}^{1} & \ldots & p_{01}^{1} q_{10}^{k} \\
p_{11}^{0} q_{11}^{0} & p_{11}^{0} q_{11}^{1} & \ldots & p_{11}^{0} q_{11}^{k} & p_{11}^{1} q_{11}^{0} & p_{11}^{1} q_{11}^{1} & \ldots & p_{11}^{1} q_{11}^{k} \\
p_{02}^{0} q_{20}^{0} & p_{02}^{0} q_{20}^{1} & \ldots & p_{02}^{0} q_{20}^{k} & p_{02}^{1} q_{20}^{0} & p_{02}^{1} q_{20}^{1} & \ldots & p_{02}^{1} q_{20}^{k} \\
\vdots & \vdots & \ddots & \vdots & \vdots & \vdots & \ddots & \vdots \\
p_{0 k}^{0} q_{k 0}^{0} & p_{0 k}^{0} q_{k 0}^{1} & \ldots & p_{0 k}^{0} q_{k 0}^{k} & p_{0 k}^{1} q_{k 0}^{0} & p_{0 k}^{1} q_{k 0}^{1} & \cdots & p_{0 k}^{1} q_{k 0}^{k} \\
p_{1 k}^{0} q_{k 1}^{0} & p_{1 k}^{0} q_{k 1}^{1} & \ldots & p_{1 k}^{0} q_{k 1}^{k} & p_{1 k}^{1} q_{k 1}^{0} & p_{1 k}^{1} q_{k 1}^{1} & \ldots & p_{1 k}^{1} q_{k 1}^{k}
\end{array}\right]
$$

In the above matrix $P(p, q)$, consider a previous round outcome $\left(s_{X}(0), s_{Y}(0)\right)$. The conditional probabilities that the speculator $X$ and the administrator $Y$ select $s_{X}(0)$ and $s_{Y}(0)$ in the current game round are $p_{00}^{0}$ and $q_{00}^{0}$, respectively. Therefore, the probability of transitioning from the previous state $\left(s_{X}(0), s_{Y}(0)\right)$ to the current state $\left(s_{X}(0), s_{Y}(0)\right)$ is $p_{00}^{0} q_{00}^{0}$. We denote by $\bar{P}=P-I$ such that

$$
\bar{P}(p, q)=\left[\begin{array}{ccccccccc}
p_{00}^{0} q_{00}^{0}-1 & p_{00}^{0} q_{00}^{1} & \ldots & p_{00}^{0} q_{00}^{k} & p_{00}^{1} q_{00}^{0} & p_{00}^{1} q_{00}^{1} & \ldots & p_{00}^{1} q_{00}^{k} \\
p_{10}^{0} q_{01}^{0} & p_{10}^{0} q_{01}^{1}-1 & \ldots & p_{10}^{0} q_{01}^{k} & p_{10}^{1} q_{01}^{0} & p_{10}^{1} q_{01}^{1} & \ldots & p_{10}^{1} q_{01}^{k} \\
p_{01}^{0} q_{10}^{0} & p_{01}^{0} q_{10}^{1} & \ldots & p_{01}^{0} q_{10}^{k} & p_{01}^{1} q_{10}^{0} & p_{01}^{1} q_{10}^{1} & \ldots & p_{01}^{1} q_{10}^{k} \\
p_{11}^{0} q_{11}^{0} & p_{11}^{0} q_{11}^{1} & \ldots & p_{11}^{0} q_{11}^{k} & p_{11}^{1} q_{11}^{0} & p_{11}^{1} q_{11}^{1} & \ldots & p_{11}^{1} q_{11}^{k} \\
p_{02}^{0} q_{20}^{0} & p_{02}^{0} q_{20}^{1} & \ldots & p_{02}^{0} q_{20}^{k} & p_{02}^{1} q_{20}^{0} & p_{02}^{1} q_{20}^{1} & \ldots & p_{02}^{1} q_{20}^{k} \\
\vdots & \vdots & \ddots & \vdots & \vdots & \vdots & \ddots & \vdots \\
p_{0 k}^{0} q_{k 0}^{0} & p_{0 k}^{0} q_{k 0}^{1} & \ldots & p_{0 k}^{0} q_{k 0}^{k} & p_{0 k}^{1} q_{k 0}^{0} & p_{0 k}^{1} q_{k 0}^{1} & \ldots & p_{0 k}^{1} q_{k 0}^{k} \\
p_{1 k}^{0} q_{k 1}^{0} & p_{1 k}^{0} q_{k 1}^{1} & \ldots & p_{1 k}^{0} q_{k 1}^{k} & p_{1 k}^{1} q_{k 1}^{0} & p_{1 k}^{1} q_{k 1}^{1} & \ldots & p_{1 k}^{1} q_{k 1}^{k}-1
\end{array}\right] .
$$


We further assume that the vector $v=\left(v_{1}, v_{2}\right.$, $\left.\ldots, v_{2(k+1)}\right)$ is the stationary vector of the state transition matrix $P$. According to the properties of a random matrix, we obtain

$$
v^{T} P=v^{T} \text { or } v^{T} \bar{P}=0 .
$$

The property of the determinant Cramer's rule, when applied to the matrix $\bar{P}$, can result in

$$
\operatorname{Adj}(\bar{P}) \bar{P}=\operatorname{det}(\bar{P}) I,
$$

where $\operatorname{Adj}(\bar{P})$ is the adjugate matrix of $\bar{P}$. As can be seen from the $P(p, q)$ matrix, we know that $p_{i j}^{0}+p_{i j}^{1}=1, q_{j i}^{0}+q_{j i}^{1}+\cdots+q_{j i}^{k}=1$, and the $P(p, q)$ matrix has the unit eigenvalue. Matrix $\bar{P}$ is singular, thus having a zero determinant. It satisfies the following condition:

$$
\operatorname{det}(\bar{P})=\operatorname{det}(\bar{P}-I)=0 .
$$

Combining equations (8)-(10), we know that

$$
\operatorname{Adj}(\bar{P}) \bar{P}=\operatorname{det}(\bar{P}) I=v^{T} \bar{P}=0 .
$$

Equation (11) implies that every row of $\bar{P}$ is proportional to the vector $v$. Suppose that the last row of $\operatorname{Adj}(\bar{P})$ is $\left(A_{1(2 k+2)}, A_{2(2 k+2)}, A_{3(2 k+2)}, \ldots, A_{(2 k+2)(2 k+2)}\right)$, which is the algebraic cofactor of the last column of $\operatorname{det}(\overline{\mathrm{P}})$. From equation (11), there exists a constant $\phi \geq 0$ that satisfies

$$
\begin{aligned}
& \phi A_{1(2 k+2)}=v_{1}, \\
& \phi A_{2(2 k+2)}=v_{2}, \\
& \phi A_{3(2 k+2)}=v_{3}, \ldots, \phi A_{(2 k+2)(2 k+2)}=v_{2 k+2} .
\end{aligned}
$$

For an arbitrary vector $f=\left(f_{1}, f_{2}, \ldots, f_{2 k+2}\right)$, the inner product of $v \cdot f$ satisfies

$$
\begin{aligned}
v \cdot f= & v_{1} \cdot f_{1}+v_{2} \cdot f_{2}+\cdots+v_{2 k+2} \cdot f_{2 k+2}=\phi A_{1(2 k+2)} \\
& \cdot f_{1}+\phi A_{2(2 k+2)} \cdot f_{2}+\cdots+\phi A_{(2 k+2)(2 k+2)} \cdot f_{2 k+2} .
\end{aligned}
$$

The Laplace transformation applied to $\operatorname{det}(\bar{P})$ generates

$$
\begin{aligned}
\operatorname{det}(\bar{P}(p, q))= & a_{1(2 k+2)} \cdot A_{1(2 k+2)}+a_{2(2 k+2)} \\
& \cdot A_{2(2 k+2)}+\cdots+a_{(2 k+2)(2 k+2)} \cdot A_{(2 k+2)(2 k+2)},
\end{aligned}
$$

where $a_{(2 k+2)(2 k+2)}$ is the last column of $\operatorname{det}(\overline{\mathrm{P}})$. If we change the last column of $\operatorname{det}(\overline{\mathrm{P}})$ to $f$, then we can further combine equations (13) and (14) to arrive at

$$
\begin{aligned}
\operatorname{det}(\bar{P}(p, q, f))=D(p, q, f)= & f_{1} \cdot A_{1(2 k+2)}+f_{2} \cdot A_{2(2 k+2)}+\cdots+f_{(2 k+2)(2 k+2)} \cdot A_{(2 k+2)(2 k+2)}, \\
v \cdot f=D(p, q, f) & =\left[\begin{array}{ccccccccc}
p_{00}^{0} q_{00}^{0}-1 & p_{00}^{0} q_{00}^{1} & \ldots & p_{00}^{0} q_{00}^{k} & p_{00}^{1} q_{00}^{0} & p_{00}^{1} q_{00}^{1} & \ldots & f_{1} \\
p_{10}^{0} q_{01}^{0} & p_{10}^{0} q_{01}^{1}-1 & \ldots & p_{10}^{0} q_{01}^{k} & p_{10}^{1} q_{01}^{0} & p_{10}^{1} q_{01}^{1} & \ldots & f_{2} \\
p_{01}^{0} q_{10}^{0} & p_{01}^{0} q_{10}^{1} & \ldots & p_{01}^{0} q_{10}^{k} & p_{01}^{1} q_{10}^{0} & p_{01}^{1} q_{10}^{1} & \ldots & f_{3} \\
p_{11}^{0} q_{11}^{0} & p_{11}^{0} q_{11}^{1} & \ldots & p_{11}^{0} q_{11}^{k} & p_{11}^{1} q_{11}^{0} & p_{11}^{1} q_{11}^{1} & \ldots & f_{4} \\
p_{02}^{0} q_{20}^{0} & p_{02}^{0} q_{20}^{1} & \ldots & p_{02}^{0} q_{20}^{k} & p_{02}^{1} q_{20}^{0} & p_{02}^{1} q_{20}^{1} & \ldots & f_{5} \\
\vdots & \vdots & \ddots & \vdots & \vdots & \vdots & \ddots & \vdots \\
p_{0 k}^{0} q_{k 0}^{0} & p_{0 k}^{0} q_{k 0}^{1} & \ldots & p_{0 k}^{0} q_{k 0}^{k} & p_{0 k}^{1} q_{k 0}^{0} & p_{0 k}^{1} q_{k 0}^{1} & \ldots & f_{2 k} \\
p_{1 k}^{0} q_{k 1}^{0} & p_{1 k}^{0} q_{k 1}^{1} & \ldots & p_{1 k}^{0} q_{k 1}^{k} & p_{1 k}^{1} q_{k 1}^{0} & p_{1 k}^{1} q_{k 1}^{1} & \ldots & f_{2 k-1}
\end{array}\right] .
\end{aligned}
$$

In the urban public management game, the inner products $v \cdot u_{X}$ and $v \cdot u_{Y}$ yield the speculator $X$ 's and the administrator $Y$ 's expected payoffs, respectively, in the stationary state. Given equation (16), the inner products $v \cdot u_{X}$ and $v \cdot u_{Y}$ are equal to the determinants of the matrix that is obtained by replacing the last column of $\bar{P}$ by $u_{X}$ and $u_{Y}$, respectively. In the stationary state, their respective normalization payoffs are then

$$
\begin{aligned}
& U_{X}=\frac{v \cdot u_{X}}{v \cdot 1}=\frac{D\left(p, q, u_{X}\right)}{D(p, q, 1)}, \\
& U_{Y}=\frac{v \cdot u_{Y}}{v \cdot 1}=\frac{D\left(p, q, u_{Y}\right)}{D(p, q, 1)},
\end{aligned}
$$

where 1 is the vector with all components equating to unity. The payoff matrix of Table 1 indicates that

$$
\begin{aligned}
u_{X}= & \left(a, a, a, \ldots, R-r_{2}(0), R-r_{2}(1), \ldots, R-r_{2}(k)\right), \\
u_{Y}= & \left(r_{1}(0), r_{1}(1)-c, \ldots, r_{1}(k)-c k, r_{2}(0)\right. \\
& \left.+r_{1}(1), \ldots, r_{2}(k)+r_{1}(k)-c k\right) .
\end{aligned}
$$

According to the properties of matrix determinants, the determinants are unchanged if we add the second column to the $k$ column of $D\left(p, q, u_{Y}\right)$ to the first column, or add the $k+n(2 \leq n \leq k-1)$ column to the $n$ column. After performing a determinant-column addition operation on $D\left(p, q, u_{Y}\right)$, the administrator $Y$ 's expected payoffs can summarized as 


$$
v \cdot u_{Y}=D\left(p, q, u_{Y}\right)=\left[\begin{array}{ccccccccccc}
p_{00}^{0}-1 & q_{00}^{1} & q_{00}^{2} & \ldots & p_{00}^{0} q_{00}^{k} & p_{00}^{1} q_{00}^{0} & p_{00}^{1} q_{00}^{1} & \ldots & r_{1}(0) \\
p_{10}^{0}-1 & q_{01}^{1}-1 & q_{01}^{2} & \ldots & p_{10}^{0} q_{01}^{k} & p_{10}^{1} q_{01}^{0} & p_{10}^{1} q_{01}^{1} & \ldots & r_{1}(1)-c \\
p_{01}^{0}-1 & q_{10}^{1} & q_{10}^{2}-1 & \ldots & p_{01}^{0} q_{10}^{k} & p_{01}^{1} q_{10}^{0} & p_{01}^{1} q_{10}^{1} & \ldots & \vdots \\
p_{11}^{0} & q_{11}^{1} & q_{11}^{2} & \ldots & p_{11}^{0} q_{11}^{k} & p_{11}^{1} q_{11}^{0} & p_{11}^{1} q_{11}^{1} & \ldots & \vdots \\
p_{02}^{0} & q_{20}^{1} & q_{20}^{2} & \ldots & p_{02}^{0} q_{20}^{k} & p_{02}^{1} q_{20}^{0} & p_{02}^{1} q_{20}^{1} & \ldots & \vdots \\
\vdots & \vdots & \vdots & \ddots & \vdots & \vdots & \vdots & \ddots & \vdots \\
p_{0 k}^{0} & q_{k 0}^{1}-1 & q_{k 0}^{2} & \ldots & p_{0 k}^{0} q_{k 0}^{k} & p_{0 k}^{1} q_{k 0}^{0} & p_{0 k}^{1} q_{k 0}^{1} & \ldots & \vdots \\
p_{1 k}^{0} & p_{k 1}^{2} & p_{k 1}^{2} & \ldots & p_{1 k}^{0} q_{k 1}^{k} & p_{1 k}^{1} q_{k 1}^{0} & p_{1 k}^{1} q_{k 1}^{1} & \ldots & \vdots
\end{array}\right] .
$$

It is noteworthy that equation (19) is a determinant whose first column $\widehat{p}=\left(p_{00}^{0}-1, p_{10}^{0}-1, p_{01}^{0}-1, \ldots, p_{1 k}^{0}\right)^{T}$ is solely determined by the speculator $X$. In contrast, any column $\hat{q}$, taken from the second column to the $k-1$ column, is under full control of the administrator $Y$. The administrator $Y$ can unilaterally enforce a linear relationship between the two players' expected payoffs such that

$$
\alpha U_{X}+\beta U_{Y}+\gamma=\frac{D\left(\widehat{p}, \widehat{q}, \alpha u_{X}+\beta u_{Y}+\gamma\right)}{D(\widehat{p}, \widehat{q}, 1)},
$$

where $\alpha, \beta$, and $\gamma$ are all constants. If the city administrator sets the probability of the supervision intensity $\hat{q}$ properly and lets it meet the following condition,

$$
\widehat{q}=\alpha u_{X}+\beta u_{Y}+\gamma
$$

then the determinant $D\left(\hat{p}, \widehat{q}, \alpha u_{X}+\beta u_{Y}+\gamma\right)$ will vanish, and a linear relationship between the two players' expected payoffs can be established such that

$$
\alpha U_{X}+\beta U_{Y}+\gamma=0 .
$$

The mixed strategy $\widehat{q}$ resulting in the linear equation (22) is called the multistrategy $\mathrm{ZD}$ strategy of a public management game. However, in contrast to the two-strategy game, the administrator $Y$ can set any column from the second to $(k-1)$ of determinant $D\left(p, q, u_{Y}\right)$ equal to $\widehat{q}$. Therefore, our model has multiple $(k-2)$ ZD strategies which are related to the number of strategies that can be chosen by the administrator $Y$ at each interactive round of the game.

3.3. The Result of the Zero-Determinant Strategies. In the iterated game, the administrator $Y$ chooses the supervision intensity $k$ from the strategy set $S_{Y}$ under the game played with the speculator $X$. Player $Y$ can make a supervision decision based on enforcing the ZD strategies to improve the urban environment management efficiency. Now, we use the probability of supervision intensity $k=1$ in the long-term iterated game as an example to analyze the results of the $\mathrm{ZD}$ strategies. This means that the administrator $Y$ sets the second column of determinant $D\left(p, q, u_{Y}\right)$ equal to $\hat{q}$, that is, $\widehat{q}=\left(q_{00}^{1}, q_{01}^{1}-1, \ldots q_{k 1}^{1}\right)$.

We define $\beta=0$ and $\widehat{q}=\alpha u_{X}+\gamma$ in equation (20). So, $Y$ needs only to play a mixed strategy satisfying the following condition:

$$
\widehat{q}=\left[\alpha\left(a, a, a, \ldots, a, R-r_{2}(0), R-r_{2}(1), \ldots, R-r_{2}(k)\right)+\gamma \cdot 1\right]^{T},
$$

which is a system of equations with $2 k$ unknowns. In this situation, the linear relationship between the two players' expected payoffs becomes subject to

$$
\begin{aligned}
\alpha U_{X}+\gamma & =D\left(p, \alpha u_{X}+\gamma, \alpha u_{X}+\gamma\right)=0, \\
U_{X} & =-\frac{\gamma}{\alpha} .
\end{aligned}
$$

Equation (24) implies that the administrator $X$ unilaterally pins down the speculator $X$ 's expected payoffs at the level of $-(\gamma / \alpha)$. The speculators can get extra expected payoffs from speculation behavior, but the administrator can set the value $-(\gamma / \alpha)$ to reduce the expected payoffs and speculation behavior.

Let the equation $\gamma=-(\alpha+\beta) \pi$ hold. Then, equation (22) satisfies

$$
\begin{aligned}
\alpha U_{X}+\beta U_{Y}-(\alpha+\beta) \pi & =0, \\
\left(U_{Y}-\pi\right) & =-\frac{\alpha}{\beta}\left(U_{X}-\pi\right), \\
\left(U_{Y}-\pi\right) & =\lambda\left(U_{X}-\pi\right),
\end{aligned}
$$

where $\pi \leq R$ and $\lambda=-(\alpha / \beta)>1$ are constants. The administrator $Y$ can guarantee his own surplus expected payoffs over the speculator $X$ 's $\lambda$-fold if $Y$ plays a mixed strategy satisfying $\widehat{q}=\theta\left[\left(u_{Y}-\pi\right)-\lambda\left(u_{X}-\pi\right)\right]$. This is a substrategy, called the $\lambda$-extortion, of ZD strategies. Given $u_{X}$ and $u_{Y}, \hat{q}$ satisfies the definition as follows:

$$
\widehat{q}=\theta\left[\left(\left(r_{1}(0), \ldots, r_{1}(k)-c k, \ldots, r_{2}(k)+r_{1}(k)-c k\right)-\pi\right)-\lambda\left(\left(a, \ldots, a, R-r_{2}(0), \ldots, R-r_{2}(k)\right)-\pi\right)\right]^{T},
$$


where a sufficiently small $\theta \geq 0$ is a free parameter to ensure the probability stay within the range of $0 \leq q_{j i}^{1} \leq 1$. Equation (25) shows that, irrespective of how speculators evade regulation, the administrator can obtain a higher expected payoff by setting the probability of the mixed strategy $s_{Y}(1)$ to the holding of equation (26).

\section{Numerical Examples}

The results of the $\mathrm{ZD}$ strategies analyzed in the previous section show that the administrator can, in theory, enforce the ZD strategy to unilaterally define a linear relationship between two players' long-term payoffs. In order to illustrate how the administrator enforces the ZD strategy and provide supportive evidence for the theoretical predictions of the model, we specifically suppose that the administrator $Y$ has three strategies available with $k=3$ and use numerical examples to fix the specific forms of the function $r_{1}(k)$ and $r_{2}(k)$. By doing this, we can get analytical results of the equations (23)-(26). Let the functions $r_{1}(k)$ and $r_{2}(k)$ in the payoff matrix take the following form:

$$
\begin{aligned}
& r_{1}(k)=\ln (m+k), \\
& r_{2}(k)=n k,
\end{aligned}
$$

where the parameters $m$ and $n$ are constants, representing scale parameters in the particular function specifications.

Looking at the second column of equation (19), together with equation (27) and equation (28), we know that the equation system (23) should meet the following list of conditions:

$$
\left\{\begin{array}{l}
q_{00}^{1}=\alpha a+\gamma \\
q_{01}^{1}-1=\alpha a+\gamma \\
q_{10}^{1}=\alpha a+\gamma \\
q_{11}^{1}=\alpha R+\gamma \\
q_{20}^{1}-1=\alpha(R-n)+\gamma \\
q_{21}^{1}=\alpha(R-2 n)+\gamma
\end{array}\right.
$$

Once the probability $q_{11}^{1}$ is identified, the analytical solution to equation (30) can be written as

$$
\left\{\begin{array}{l}
q_{00}^{1}=0 \\
q_{01}^{1}=1 \\
q_{10}^{1}=0 \\
q_{20}^{1}=\frac{q_{11}^{1}}{R-a}(R-n-a)+1 \\
q_{21}^{1}=\frac{q_{11}^{1}}{R-a}(R-2 n-a)
\end{array}\right.
$$

in which the parameters $R, n$, and $a$ are constant terms. Equation (30) hence supports the theoretical results of the
ZD strategies in the mixed strategy game. This result shows that the administrator can decide the supervision intensity $k$ according to the probability specified by equation (30) and can unilaterally pin down the speculator's expected payoffs. For example, a probability $q_{01}^{1}=1$ means that the supervision intensity $s_{Y}(1)$ must be selected by the administrator for the current game round, conditional on the previous game outcome $\left(s_{Y}(0), s_{X}(1)\right)$, which is similar to the tit-for-tat (TFT) strategy [12]. In this situation, the speculator $X$ 's expected payoffs are fixed at

$$
U_{X}=\frac{v \cdot u_{X}}{v \cdot 1}=-\frac{\gamma}{\alpha}=a .
$$

Equation (31) shows that the administrator can unilaterally determine the expected payoffs of the speculators as the opportunity cost of legal behavior $a$. However, the result is independent of the supervision intensity $k$ and the specific functional forms of $r_{1}(k)$ and $r_{2}(k)$. In real life, speculation behavior is often reduced during a campaign of high-intensity enforcement. However, once extraordinary measures are relaxed, the speculation behavior will probably return to its previous high level. This means that the administrator's supervision mode, which is characterized by an increase in supervision and punishment, not only increases the cost of management but also fails to reduce speculation in a fundamental sense.

The administrator should enforce the ZD strategy and reduce the speculator's opportunity cost $a$ of legal behaviors. Therefore, the speculator $X$ 's expected payoffs will decrease, and speculators will change their behavior from speculation to cooperation. The public administrator must change the management model from stronger supervision to improving the public management environment and incorporating better service. For example, public administrators should plan urban parking spaces reasonably and reduce parking costs for illegal parking behavior performed by drivers. The administrator should strengthen the government's planning to legalize the activities of peddlers and scientifically plan the number, location, and business hours for peddlers to avoid adversely affecting the daily life of other urban residents. These similar measures, which represent upgrades to administrators' services, improve the public environment and can effectively reduce the opportunity cost $a$ of law-abiding behavior. The numerical example of our model explains again that the ZD strategy and a change in the administrator's management model can promote the emergence of cooperative behavior and efficiency of public management decision-making.

Regarding to the second column of equation (19) and the constraints in equations (27)-(29), the administrator enforces the strategy $\hat{q}=\theta\left[\left(u_{Y}-\pi\right)-\lambda\left(u_{X}-\pi\right)\right]$ that satisfies

$$
\left\{\begin{array}{l}
q_{00}^{1}=\theta[(\ln (m)-\pi)-\lambda(a-\pi)], \\
q_{01}^{1}-1=\theta[(\ln (m+1)-c-\pi)-\lambda(a-\pi)], \\
q_{10}^{1}=\theta[(\ln (m+2)-2 c-\pi)-\lambda(a-\pi)], \\
q_{11}^{1}=\theta[(\ln (m)-\pi)-\lambda(R-\pi)], \\
q_{20}^{1}-1=\theta[(n-c+\ln (m+1)-\pi)-\lambda(R-n-\pi)], \\
q_{21}^{1}=\theta[(2 n-2 c+\ln (m+2)-\pi)-\lambda(R-2 n-\pi)],
\end{array}\right.
$$


where the parameters $a, R, \pi, n$, and $m$ all take constant values. As long as $\theta$ is small enough, it is guaranteed that $0 \leq q_{j i}^{1} \leq 1$.

Under the extortion of the ZD strategy $\hat{q}=\theta\left[\left(u_{Y}-\pi\right)\right.$ $\left.-\lambda\left(u_{X}-\pi\right)\right]$, the administrator $Y$ 's expected payoffs depend on $X$ 's strategy probability, and the speculator $X$ 's expected payoffs are maximized when $X$ is fully cooperative, with $\widehat{p}=$ $\left(p_{00}^{0}, p_{01}^{0}, p_{10}^{0}, p_{11}^{0}, p_{20}^{0}, p_{21}^{0}\right)=(1,1,1,1,1,1)$. Equation (25) shows that the rational fully cooperative behavior of the speculator $X$ will maximize both the players' expected payoffs simultaneously. The result shows that the extortion of strategy will force speculators to choose the strategy of full cooperation (law-abiding behaviors), which is the ideal outcome of the public management game for the administrator. If the probability supervision intensity decision satisfies equation (32), the expected payoffs of both players are, respectively,

$$
\begin{aligned}
& U_{X}=R-2 n, \\
& U_{Y}=\pi-\lambda(\pi-R+2 n) .
\end{aligned}
$$

Equations (33) and (34) together reveal that the surplus expected payoff $U_{Y}-\pi$ is a $\lambda$-fold version of $U_{X}-\pi$ when the speculator $X$ is fully rational and behaves in a completely cooperative way.

Irrespective of how the speculator chooses the probability of cooperation and defection, the administrator can determine that his surplus payoffs will exceed those of the speculator. However, the size of the surplus payoff $U_{Y}-\pi$ of the administrator depends on the strategy of the speculator. Therefore, the administrator should guide speculators to adopt full cooperation that maximizes the expected payoffs of both players and improve the decision-making efficiency through the emergence of cooperative behavior. For example, business regulators should reduce the fees and the complexity of approval procedures, and the number of businesses operating without a license will decline. The urban public manager should lower the threshold of access and subsidize the early stage of legal operation for the peddler.

\section{Conclusion}

The ZD strategies for two-strategy games have changed our understanding of game theory, the $\mathrm{ZD}$ approach allows a player to unilaterally determine the expected payoffs of the opponent and have stronger control ability than traditional strategies, such as the TFT, generous TFT (GTFT), and winstay-lose-shift (WSLS) strategies. There are many interaction phenomena involving regulation and speculation in politics, economics, society, and life. Group decision-making exists widely in society $[37,38]$. Selfish defection behavior will lead to the tragedy of the commons and reduce the welfare of the whole society. Therefore, improving cooperative behavior is of great significance in solving social difficulties.

The supervision intensity decision is an important problem in policy implementation in public management. For more general application and research of ZD strategies, we have taken a step beyond a two-strategy game to a multistrategy game, with the iterated urban public management game as the scenario. We prove the existence of ZD strategies for multistrategy games by constructing a direct extension of Press and Dyson's method [3]. Moreover, the conditions of the multistrategy, as well as those of the extortion strategy, are carefully discussed. The results confirm that multiple ZD strategies are different from the two-player games, and the quantity is related to the number of strategies for the player in each round of the game. The administrator can unilaterally control the expected payoffs of speculators and promote the emergence of cooperative behaviors, by enforcing the ZD strategies to determine the level of supervision intensity in policy implementation.

\section{Data Availability}

This is a theoretical article with no data available.

\section{Conflicts of Interest}

The authors declare that there are no conflicts of interest regarding the publication of this paper.

\section{Acknowledgments}

This work was supported by the National Natural Science Foundation of China (Grant nos. 71801139 and 71571119), School Level Gold Course <INVESTMENTS> (Grant no. SBS-2019-XJJK-04), and the Young Teacher Training Funding Program by Shanghai Education Commission (Grant no. AG-14007).

\section{References}

[1] J. F. Nash, "Equilibrium points in N-person games," Proceedings of the National Academy of Sciences, vol. 36, no. 1, pp. 48-49, 1950.

[2] J. M. Smith and G. R. Price, "The logic of animal conflict," Nature, vol. 246, no. 5427, pp. 15-18, 1973.

[3] W. H. Press and F. J. Dyson, "Iterated Prisoner's Dilemma contains strategies that dominate any evolutionary opponent," Proceedings of the National Academy of Sciences, vol. 109, no. 26, pp. 10409-10413, 2012.

[4] L. Pan, D. Hao, Z. Rong, and T. Zhou, "Zero-determinant strategies in iterated public goods game," Scientific Reports, vol. 5, no. 1, Article ID 13096, 2015.

[5] Y. Dai, Y. Qi, L. Li, B. Wang, and H. Gao, "A dynamic pricing scheme for electric vehicle in photovoltaic charging station based on Stackelberg game considering user satisfaction," Computers \& Industrial Engineering, vol. 154, Article ID 107117, 2021.

[6] Y. M. Dai, Y. Qi, and L. Li, “A real-time pricing scheme with advertisement competition based on multi-leader-multi-follower game in smart community," Asia-Pacific Journal of Operational Research, vol. 38, 2021.

[7] Y. M. Dai, X. L. Sun, and Y. Qi, "A real-time, personalized consumption-based pricing scheme for the consumptions of traditional and renewable energies," Renewable Energy, vol. 180, pp. 452-466, 2020.

[8] X. Zhan, C. W.-H. Lo, and S.-Y. Tang, "Contextual changes and environmental policy implementation: a longitudinal 
study of street-level bureaucrats in guangzhou, China," Journal of Public Administration Research and Theory, vol. 24, no. 4, pp. 1005-1035, 2014.

[9] R. Ran, "Perverse incentive structure and policy implementation gap in China's local environmental politics," Journal of Environmental Policy and Planning, vol. 15, no. 1, pp. 17-39, 2014.

[10] S. B. M. Kraak, "Exploring the 'public goods game' model to overcome the Tragedy of the Commons in fisheries management," Fish and Fisheries, vol. 12, no. 1, pp. 18-33, 2011.

[11] R. Cooper, D. V. Dejong, and R. Forsythe, "Cooperation without reputation: experimental evidence from prisoner's Dilemma games," Games and Economic Behavior, vol. 12, no. 12, pp. 187-218, 1992.

[12] R. Axelrod and W. D. Hamilton, "The evolution of cooperation," Science, vol. 211, no. 4489, pp. 1390-1396, 1981.

[13] R. L. Trivers, "The evolution of reciprocal altruism," The Quarterly Review of Biology, vol. 46, no. 1, pp. 35-57, 1971.

[14] A. Dreber, D. G. Rand, D. Fudenberg, and M. A. Nowak, "Winners don't punish," Nature, vol. 452, no. 7185, pp. 348-351, 2008.

[15] J.-J. Wu, B.-Y. Zhang, Z.-X. Zhou et al., "Costly punishment does not always increase cooperation," Proceedings of the National Academy of Sciences, vol. 106, no. 41, pp. 17448-17451, 2009.

[16] E. Fehr and B. Rockenbach, "Detrimental effects of sanctions on human altruism," Nature, vol. 422, no. 6928, pp. 137-140, 2003.

[17] J. Schwartz, "The impact of state capacity on enforcement of environmental policies: the case of China," The Journal of Environment \& Development, vol. 12, no. 1, p. 50, 2003.

[18] M. S. Hamm and J. L. Schrink, "The conditions of effective implementation," Criminal Justice and Behavior, vol. 16, no. 2, pp. 166-182, 1989.

[19] G. Kostka, Barrier to the Implementation of Environmental Policies at the Local Level in China, 7016 pages, World Bank Policy Research Working Paper, Washington, DC, USA, 2017.

[20] N. Liu, B. Van Rooij, and C. W.-H. Lo, "Beyond deterrent enforcement styles: behavioural intuitions of Chinese environmental law enforcement agents in a context of challenging inspections," Public Administration, vol. 96, no. 3, pp. 497-512, 2018.

[21] X. Zhao and L. Ortolano, "Implementing China's national energy conservation policies at state-owned electric power generation plants," Energy Policy, vol. 38, no. 10, pp. 6293-6306, 2012.

[22] G. Kostka and A. P. J. Mol, "Implementation and participation in China's local environmental politics: challenges and innovations," Journal of Environmental Policy and Planning, vol. 15, no. 1, pp. 3-16, 2013.

[23] R. A. W. Rhodes and D. Marsh, "New directions in the study of policy networks," European Journal of Political Research, vol. 21, no. 1-2, pp. 181-205, 2010.

[24] S. Togawa, "The relationship between governance and new public management: policy network perspective and new institutional perspective," Yamanashi Gakuin Law Review, vol. 56, 2006.

[25] L. Kitchen, "Environmental policy and the differentiation of rural space: an actor-network perspective," Journal of Environmental Policy and Planning, vol. 2, no. 2, pp. 135-147, 2020.

[26] F. Xu, M. Tian, J. Yang, and G. Xu, "Does environmental inspection led by the central government improve the air quality in China? The moderating role of public engagement," Sustainability, vol. 12, no. 8, p. 3316, 2020.

[27] F. Meng, Z. Chen, and J. Wu, "How are anti-air pollution policies implemented? A network analysis of campaign-style enforcement in China," Sustainability, vol. 11, no. 2, p. 340, 2019.

[28] N. N. Liu, C. W.-H. Lo, X. Zhan, and W. Wang, "Campaignstyle enforcement and regulatory compliance," Public Administration Review, vol. 75, no. 1, pp. 85-95, 2015.

[29] G. Kostka and C. Zhang, "Tightening the grip: environmental governance under Xi Jinping," Environmental Politics, vol. 27, no. 5, pp. 769-781, 2018.

[30] G. Xiong and Y. Hou, "The game model with emotional factors of public and media in public emergencies management engineering," Systems Engineering Procedia, vol. 5, pp. 228-233, 2012.

[31] J. M. Tavares, G. de Vargas Mores, and E. L. Tomazzoni, "Where to invest in touristic promotion? Game theory as a decision instrument for the public sector," Tourism Economics, vol. 21, no. 4, pp. 775-789, 2015.

[32] L. Sun, Z. Gao, and Y. A. Wang, "Stackelberg game management model of the urban public transport," Journal of Industrial and Management Optimization, vol. 8, no. 2, pp. 507-520, 2017.

[33] C. Adami and A. Hintze, "Evolutionary instability of Zero Determinant strategies demonstrates that winning isn't everything," Nature Communications, vol. 4, no. 4, p. 2193, 2012.

[34] C. Hilbe, M. A. Nowak, and K. Sigmund, "Evolution of extortion in iterated prisoner's Dilemma games," Proceedings of the National Academy of Sciences, vol. 110, no. 17, pp. 6913-6918, 2013.

[35] A. J. Stewart and J. B. Plotkin, "From extortion to generosity, evolution in the Iterated Prisoner's Dilemma," Proceedings of the National Academy of Sciences, vol. 110, no. 38, pp. 15348-15353, 2013.

[36] A. Szolnoki and M. Perc, "Evolution of extortion in structured populations," Physical review. E, Statistical, nonlinear, and soft matter physics, vol. 89, Article ID 022804, 2014.

[37] Y. Ji, X. W. Jin, Z. S. Xu, and S. J. Qu, "A mixed 0-1 programming approach for multiple attribute strategic weight manipulation based on uncertainty theory," Journal of Intelligent and Fuzzy Systems, vol. 16, 2021.

[38] Y. Ji, H. H. Li, and H. J. Zhang, "Risk-averse two-stage stochastic minimum cost consensus models with asymmetric adjustment cost," Group Decision and Negotiation, vol. 30, 2021. 